\title{
THE ROLE OF TRACTION THERAPY IN THE TREATMENT OF PAIN SYNDROME AND THE PSYCHO-EMOTIONAL STATE OF THE PATIENTS WITH LUMBAR DISC DISEASE
}

\author{
Mariyana Mihaylova ${ }^{1}$, Ilia Todorov' ${ }^{1}$, Silviya Filkova ${ }^{2}$, Dafina Bacheva ${ }^{1}$ \\ ${ }^{1}$ Department of Physiotherapy, Rehabilitation, Thalassotherapy, Occupational Diseases \\ and Disaster Medicine, Faculty of Medicine, Medical University of Varna, Bulgaria \\ ${ }^{2}$ TRS Rehabilitation Therapist, Medical College, Medical University of Varna, Bulgaria
}

\begin{abstract}
INTRODUCTION: Traction therapy as a method for treatment of the spinal diseases has been known to mankind since Hippocrates. Its abilities to target the main symptoms in patients with lumbar disc disease are still an object of discussion.

AIM: To analyze the effect of intermittent traction therapy in targeting pain syndrome and the psychoemotional state of the patients with lumbar disc disease.

MATERIALS AND METHODS: A total of 122 patients suffering from lumbar disc disease were examined and 100 of them corresponded to the criteria and took part in the research. The control group (CG) included 50 patients, treated with basic therapy low-frequency electromagnetic field (LFMF) and interferential therapy - bipolar technique (BPI). The experimental group (EG) included 50 patients treated with basic therapy and intermittent traction therapy. To track the effects of the treatment, we used an evaluation of the static and dynamic pain, an evaluation of the psycho-emotional state through the WAM (well-being, activity, mood) test, done before, right after and at the end of the treatment as well as 1 and 3 months after the treatment.

RESULTS: Both methodologies have a pain-reducing effect and help the improvement of the psycho-emotional state of the patients. The results have a high statistical importance $(\mathbf{p}<0.001)$ for both groups when it comes to reduction of the pain syndrome and the indicators of the psycho-functional testing. When comparing EG and CG the difference is also statistically significant $(p<0.001)$ with the EG being superior.

CONCLUSION: Traction therapy, conducted following all rules of the good medical practice is well tolerated by the patients suffering from lumbar disc disease and contributes to the improvement of their psychoemotional state.
\end{abstract}

Keywords: lumbar disc disease, psycho-emotional state, intermittent traction therapy

Address for correspondence:

Mariyana Mihaylova

Department of Physiotherapy, Rehabilitation,

Thalassotherapy, Occupational Diseases and Disaster Medicine

St. Marina University Hospital

$1 \mathrm{Hr}$. Smirnenski Blvd

Varna 9000

e-mail:m.mihailova96@abv.bg

Received: March 1, 2017

Accepted: May 15, 2017

\section{INTRODUCTION}

The chronic pathology of the spine leads to long disability, and a development of physical, psychological and social disadaptation.

The main task of the rehabilitation program is to contribute to the full recovery of the patient in physical, psycho-emotional and social aspect as well as to help improve the quality of life. 
Mariyana Mihaylova, Ilia Todorov, Silviya Filkova et al.

Pain syndrome is one of the leading vertebral diseases. Pain is a valuable diagnostic indicator but it does not always match the seriousness of the diseases. The perception of pain depends heavily on the psycho-emotional state of the individual. More and more academics claim that contributing factors to the first pain complaints are the psychological and physical aspects of the working environment (6). According to Gatchel (2008) that is the main reason for chronical lower back pain in relatively young people - between 30 and 50 years of age. In 2010, Sardá et al. found an existence of a significant link between the changes in the psychological status and chronic lower back pain. Pain as a phenomenon has many dimensions linked to the emotions and behavior. Lower back pain generates various levels of limitations in the daily activities and the quality of sleep (10). Those sleep disturbances and motor activity limitations have a direct impact over the behavior of the patients $(9,11)$. The functional state characterizes the effective side of activity or behavior of the individual. In other words, this is an aspect of the abilities of the individual to conduct certain activities.

Without a doubt the psychological factors have a significant role in the development and chronification of the neurological occurrences in lumbar disc disease. Psycho-emotional disadaptation and the individual reaction to the disease have a key role. Stress leads to change in the pain threshold and via segmental regulation of the reflex activity in the spinal and brain system affects the vertebral-neurological pathology (8).

\section{AIM}

To research the effect of traction therapy in targeting pain syndrome and the psycho-emotional state of the patients

\section{MATERIALS AND METHODS}

A total of 122 patients suffering from lumbar disc disease were examined and 100 of them corresponded to the criteria and took part in the research.

The main criteria for selecting the participants in the study for both groups were: age between 18 and 55 years; presence of lower back pain; proven degeneration of the intervertebral discs in the lumbar region - disc protrusion and herniation; ex- istence of the disease for less than 2 years; individuals who have not undergone surgical treatment.

The main criteria for excluding participants from the study for both groups were: individuals younger than 18; individuals with a pacemaker, neoplastic, infectious, etc. diseases or conditions that are contraindications to conducting a physical therapy; presence of indications for serious spinal pathology with tumors, compression fractures, infections, serious arthritic changes (bone bridges) etc.; presence of symptoms for serious root compression - Lasegue under $30^{\circ}$; weakened reflexes and sensitivity, paralysis and paresis; pregnancy; cardiac rhythms pathology; spinal surgery into the lumbar region.

Through random selection, the patients were divided into two groups each containing 50 members - experimental (EG) and control (CG). The basic treatment included: low frequency magnetic therapy (LFMF) and bipolar interference (BPI).

The CG was treated with basic therapy (LFMF and BPI) and the EG was treated with basic therapy and intermittent traction therapy. In EG the physical program was conducted once a day in the following order:

$\diamond$ Magnetic therapy with parameters $16000 \mathrm{~A} / \mathrm{m}$, $1 \mathrm{~Hz}, 0.2 \mathrm{~s}, 15-20 \mathrm{~min} ., 10$ procedures;

$\diamond$ BPI in a paravertebral positioning of the electrodes at a level (L1-S1) with parameters 90$130 \mathrm{~Hz}-15 \mathrm{~min}$;

$\diamond$ Intermittent extension therapy - duration 20 min.;

$\diamond$ The rest of the patients were in Williams' position (lying down, the legs lifted up on a stool, folded in $90^{\circ}$ just like in a sit-up position) aiming to transform the lumbar lordosis into cifosis and removing the tension from the lumbar spine (18);

$\diamond$ The procedure ended with putting an orthopaedic lumbar corset;

$\diamond$ Number of procedures: 10 .

The pulling power is determined by the severity of the degenerative changes and pain syndrome, the stage of the disease and the patient's weight. Extensive treatment is conducted in intermittent mode, which reduces significantly the risk of development of spinal instability. 
The stages of pain are assessed using the Borg scale $(4,5)$ and the modified scale of Merl d'Aubigne (3).

For assessment of the psycho-emotional state the WAM test - well-being, activity and mood, was used $(1,2)$.

\section{STATISTICS}

The gathered data was processed through the statistical software IBM SPSS for Windows, v. 19.0 using the following statistical methods:

Descriptive statistics:

$\diamond$ frequency analysis of the quality variables - calculation of absolute and relative frequencies;

$\diamond$ variation analysis of the quantitative variables - calculation of mean, standard deviation, standard error of the mean, 95\% confidence level;

$\diamond$ graphic method to visualize the results;

$\diamond$ correlation analysis.

For statistically credible are accepted differences with a significance level $\mathrm{p}<0.05$.

\section{RESULTS}

The average age of the patients in the experimental group was $38.9 \pm 1.21$ years and the control group had an average age of $39.54 \pm 1.44$ years. Both groups included mainly patients between 30 and 40 years of age. In EG the male to female ratio was 5644, while in CG it was 52-48.

Taking into account the main role of the pain syndrome for the spread of vertebral neurological symptoms, we assessed the intensity of the pain using the Borg scale for static pain and the modified scale of Merl d'Aubigne for dynamic pain. We chose this more detailed method to differentiate the two types of pain, proceeding from the key role that the spine has, both for the static of the body and its motor activity, considering the better accuracy of the pain assessment, despite the subjective factors.

In the analysis of the results from the Borg scale we saw the following relations:

before the beginning of the treatment the average estimates from the Borg scale were very close to each other, because of the random selection of the two groups (Fig. 1).

The comparison of the average estimates right after the treatment, 1 and 3 months after the treat-

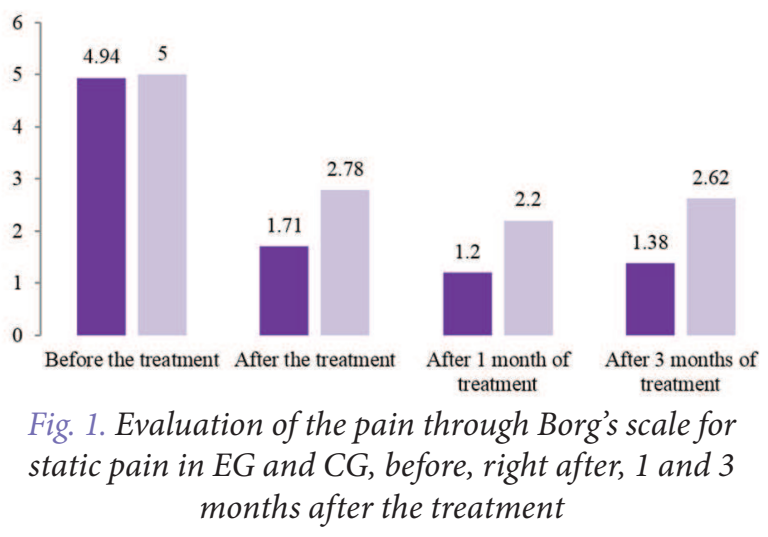

ment through paired samples t-test between the separate assessments in the groups found a significant difference $(\mathrm{p}<0.001)$. The experimental group demonstrated an advantage in every comparison.

Comparison of the results of the modified scale of Merl d'Aubigne before, right after, 1 and 3 months after the treatment ended in the two groups (Fig. 2):

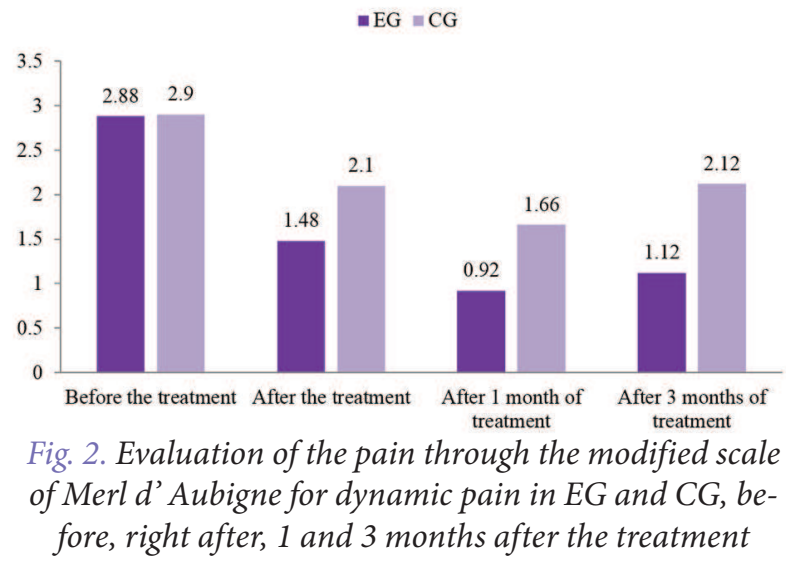

When analyzing the data from the assessment of the average values of the dynamic pain through the modified scale of Merl d'Aubigne in both groups and between them, we found a significant difference in all indicators $(\mathrm{p}<0.001)$ but when comparing the final result of the average values in the two groups respectively 1.12 for EG and 2.12 for CG, the result of the experimental group was superior to the one of the control group with a whole point, thus proving the better pain-reducing feature of extension therapy.

Comparison analysis of the results from the WAM test in EG and CG showed a positive dynam- 
Mariyana Mihaylova, Ilia Todorov, Silviya Filkova et al.

ic in the tracked psychological indicators in both groups.

After the treatment ended, both groups showed a statistically significant improvement in all three examined indicators (Fig. 3,4,5), lasting until the end of the first month. The trend of maintaining the results differed significantly after the third month and statistically significant differences could be seen in all three indicators.

The average value of the well-being indicator was 5.31 in the experimental group and 4.91 in the control group (Fig. 3).

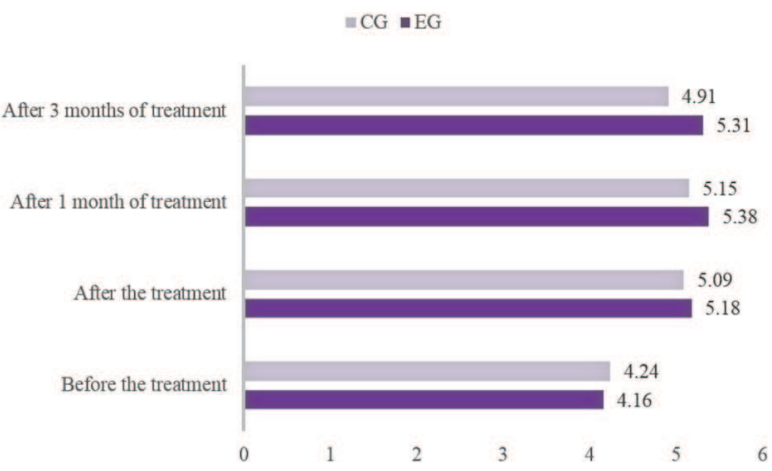

Fig. 3. Change of the well-being indicator in EG and $C G$, before, right after, 1 and 3 months after the treatment

The activity indicator showed average values of 5.58 and 4.71 for the experimental and control groups, respectively (Fig.4).

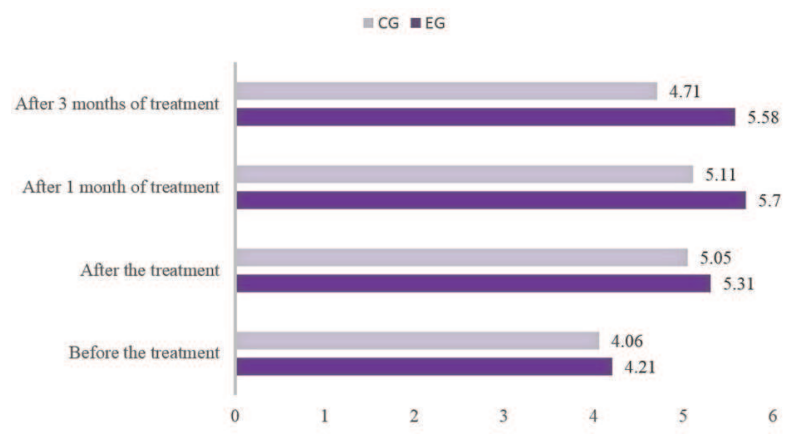

Fig. 4. Change of the activity indicator in EG and CG, before, right after, 1 and 3 months after the treatment

The average results for the mood indicators were 5.37 and 4.72 for EG and CG and the difference between the two groups was statistically significant in all comparisons $(\mathrm{p}<0.001)$ (Fig. 5).

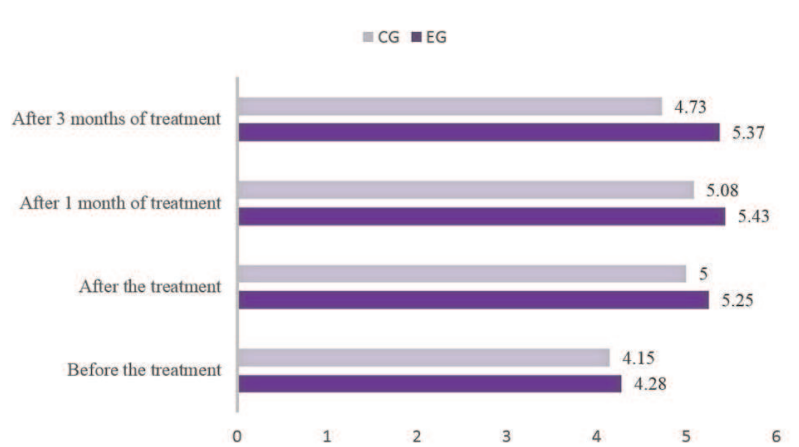

Fig. 5. Change of the mood indicator in EG and CG, before, right after, 1 and 3 months after the treatment

The trend of maintaining the results, however, differs noticeably after the third month, when statistically significant differences in the three indicators can be observed showing the superiority of EG (Fig. $3,4,5)$.

The correlation analysis of the WAM test and the modified scale of Merl d'Aubigne showed a moderate to significant correlation in a reversed manner between the two instruments. Before the treatment $\mathrm{r}=-0.456(\mathrm{p}<0.001)$, right after it $\mathrm{r}=-0.484(\mathrm{p}<0.001)$, at the end of the first month after the therapy $\mathrm{r}=$ $0.522(\mathrm{p}<0.001)$ and at the end of the tracked period $\mathrm{r}=-0.503(\mathrm{p}<0.001)$. When there's a reduction in the values of the subjective evaluation of the pain, an increase in the average value of the common indicator for evaluation of the psycho-emotional state in WAM test can be observed.

\section{DISCUSSION}

Based on the conducted study we found that alongside the abnormal neurological status in the majority of the patients, there were changes in their psycho-emotional state consisting of decreased well-being, bad mood and decreased physical activity.

The patients with prominent pain syndrome also had prominent disturbances in their psycho-emotional state. There was a correlation between the WAM indicators and those from the tests for static and dynamic pain. The patients with low indicators in WAM had higher assessments in the pain scales. According to the results from the correlation analysis of the subjective evaluation of the pain through the scale of Merl d'Aubigne and the results from WAM, a moderate to significant correlation in a reversed manner between the two instruments in all four stages of our study $(\mathrm{p}<0.001)$ was found. Analysing those results showed 
The Role of Traction Therapy in the Treatment of Pain Syndrome and the Psycho-Emotional State of the Patients with Lumbar Disc...

the superior corrective impact of the complex methodology with included traction therapy in regard to the main indicators of the psycho-functional state of the patients. In EG, the results maintained their significance up to the third month after the treatment. In CG a noticeable improvement can also be seen. It remained unaltered up to the end of the first month and then declined. These results are due to the better effect of the experimental methodology in relation to the decrease of the dynamic pain, which lead to recovery of the motor activity and also related to the long-lasting of the psycho-emotional reserves. Our results demonstrating the potential of traction therapy in targeting pain syndrome matched those of other authors $(13,14)$.

In conjunction with the fact that temotional parameters are related to the pain level, it is very important to reduce the pain with a maximum reduction of the risk of relapses.. The pain in all its aspects is a main factor leading to reduction of the quality of life of the patient. The elimination of the pain syndrome is a key task of every treatment. This requires a proper therapeutic approach and methodology that gives better results.

\section{CONCLUSION}

The results confirmed the contribution of traction therapy in correcting the psycho-emotional state of the patients. The main indicators of WAM - wellbeing, activity and mood in EG reached values closer to the norm and maintained that up to the third month after the therapy.

Traction therapy, conducted following all rules of the good medical practice is well tolerated by the patients and contributes to the improvement of their psycho-emotional state.

\section{REFERENCES}

1. Ivanov I. Methods of examination of the functional states. Series of chosen methods of the psychological and pedagogical diagnostics, vol.1. Shumen:Aksios; 1999 (in Bulgarian).

2. Leonova A. Psychodiagnostics of the human's functional state. Moscow; 1984. p. 200.

3. Todorov, T. Research of the effect of the manual therapy in cervicocranial and cervical lock, dissertation of applicant in medical sciences; 1989.

4. Borg G. A category scale with ratio properties for intermodal and interindividual comparisons. In:
Geisslar HG, Petzold P, editors. Psychophysical judgment dnd the process of perception. Berlin: VEB Deuticher Verlang der Wissenschaften; 1982. pp 25-34.

5. Borg G, Borg E. A new generation of scaling methods: level-anchored ratio scaling. Psychologica. 2001. In press. (Boos N 2010)

6. Boos N, Semmer N, Elfering A, Schade V, Gal I, Zanetti M, et al. Natural history of individuals with asymptomatic disc abnormalities in magnetic resonance imaging: predictors of low back pain-related medical consultation and work incapacity. Spine (Phila Pa 1976). 2000;25(12):1484-92.

7. Gatchel RJ, Rollings KH. Evidence-informed management of chronic low back pain with cognitive behavioral therapy.Spine J. 2008;8(1):40-4. doi: 10.1016/j.spinee.2007.10.007

8. Hoffman BM, Papas RK, Chatkoff DK, Kerns RD. Meta-analysis of psychological interventions for chronic low back pain. Health Psychol. 2007;26:1-9. doi: 10.1037/0278-6133.26.1.1

9. Kindermans HPJ, Huijnen IPJ, Goossens MEJB et al. "Being" in pain: the role of self-discrepancies in the emotional experience and activity patterns of patients with chronic low back pain. Pain. 2011;152(2):403-9. doi: 10.1016/j.pain.2010.11.009

10. Marin R, Cyhan T, Miklos W. Sleep disturbance in patients with chronic low back pain. Am J Phys Med Rehabil. 2006;85(5):430-5. doi: 10.1097/01. phm.0000214259.06380.79

11. Fine PG. Long-term consequences of chronic pain: mounting evidence for pain as a neurological disease and parallels with other chronic disease states..Pain Med. 2011;12(7): 996-1004. doi: 10.1111/j.1526-4637.2011.01187.x

12. Sardá J Jr J, Kupek E, Cruz RM, Aspectos psicológicos associados lombalgia e à lombociática. R Ci Hum. 2000;18(28):51-60. doi: 10.5007/\%25x

13. Beattie PF, Nelson RM, Michener LA, Cammarata J, Donley J. Outcomes after a prone lumbar traction protocol for patients with activity-limiting low back pain: a prospective case series study. Arch Phys Med Rehabil. 2008;89: 269-74. doi: 10.1016/j. apmr.2007.06.778

14. Diab AA, Moustafa IM. Lumbar lordosis rehabilitation for pain and lumbar segmental motion in chronic mechanical low back pain: a randomized trial. J Manipulative Physiol Ther.2012;35: 246-53. doi: 10.1016/j.jmpt.2012.04.021 